\title{
Clozapine Metabolism is Associated With Absolute Neutrophil Count in Individuals With Treatment-Resistant Schizophrenia
}

Isabella R. Willcocks ${ }^{1}$, Sophie E. Legge ${ }^{1}$, Mariana Nalmpanti ${ }^{1}$, Lucy Mazzeo ${ }^{2}$, Adrian King ${ }^{3}$, John Jansen ${ }^{4}$, Marinka Helthuis ${ }^{4}$, Michael J. Owen ${ }^{1}$, Michael C. O'Donovan ${ }^{1}$, James T. R. Walters ${ }^{1}$ and Antonio F. Pardiñas ${ }^{1 *}$

${ }^{1}$ MRC Centre for Neuropsychiatric Genetics and Genomics, Division of Psychological Medicine and Clinical Neurosciences, School of Medicine, Cardiff University, Cardiff, United Kingdom, ${ }^{2}$ Hafan y Coed Mental Health Unit, University Hospital of Llandough, Cardiff, United Kingdom, ${ }^{3}$ Magna Laboratories Ltd., Ross-on-Wye, United Kingdom, ${ }^{4}$ Leyden Delta B.V., Nijmegen, Netherlands

\section{OPEN ACCESS}

Edited by:

Moneeza Kalhan Siddiqui, University of Dundee, United Kingdom

Reviewed by:

$X i W u$,

Shanghai Jiao Tong University, China Claudia Pisanu,

University of Cagliari, Italy

*Correspondence:

Antonio F. Pardiñas

PardinasA@cardiff.ac.uk

Specialty section: This article was submitted to

Pharmacogenetics and

Pharmacogenomics,

a section of the journal

Frontiers in Pharmacology

Received: 26 January 2021

Accepted: 17 March 2021

Published: 16 April 2021

Citation:

Willcocks IR, Legge SE, Nalmpanti M,

Mazzeo L, King A, Jansen J,

Helthuis M, Owen MJ, O'Donovan MC,

Walters JTR and Pardiñas AF (2021)

Clozapine Metabolism is Associated

With Absolute Neutrophil Count in

Individuals With Treatment-

Resistant Schizophrenia.

Front. Pharmacol. 12:658734.

doi: 10.3389/fphar.2021.658734
Up to one-third of those with schizophrenia fail to respond to standard antipsychotics and are considered to have treatment-resistant schizophrenia, a condition for which clozapine is the only evidence-based medication. While up to $60 \%$ of treated individuals obtain therapeutic benefits from clozapine, it is currently underprescribed worldwide, partly because of concerns related to its broad adverse effect profile. In particular, the potential effects of clozapine on the immune system have gained relevance after a recent study showed that drug plasma concentrations were inversely correlated with neutrophil counts in individuals routinely undergoing treatment. Seeking to investigate this relationship in more detail, we extracted metabolic, immune, and genetic data from a UK cohort of long-term clozapine users linked to a clozapine monitoring service, CLOZUK2 $(N=208)$. Whilst a correlation analysis was compatible with the original results, a multiple linear regression accounting for dose and other confounding factors additionally allowed us to estimate the decrease in absolute neutrophil counts to approximately $141 \mathrm{cells} / \mathrm{mm}^{3}$ for every $0.1 \mathrm{mg} / \mathrm{L}$ increase in clozapine concentration. However, this association was attenuated after controlling for the metabolic ratio between clozapine and its main metabolite, norclozapine, which was itself negatively associated with neutrophil concentrations. Further analyses revealed that these relationships are likely moderated by genetic factors, as three pharmacogenomic SNPs previously associated to norclozapine plasma concentrations and the metabolic ratio (rs61750900, rs2011425 and rs1126545) were shown to be independently associated with a variation in neutrophil counts of about 400 cells $/ \mathrm{mm}^{3}$ per effect allele. Such results are compatible with an effect of norclozapine, but not necessarily clozapine, on immune cell counts, and highlight the need for further investigations into the potential role of genetic determinants of clozapine pharmacokinetics in the occurrence of adverse effects during treatment.

Keywords: clozapine, norclozapine, metabolic ratio, genetics, treatment-resistant schizophrenia 


\section{INTRODUCTION}

Schizophrenia is typically a severe, chronic neuropsychiatric disorder affecting an estimated $0.7 \%$ of the global population (McGrath et al., 2008). Although antipsychotic medications are generally effective, $20-30 \%$ of patients are classified as having 'Treatment-Resistant' Schizophrenia (TRS), defined as a lack of symptomatic relief following trials of at least two antipsychotics at an adequate dose for a reasonable time (Howes et al., 2017). For these individuals, treatment options are often limited to a single medication; clozapine, the first atypical antipsychotic drug developed, and the only drug currently available with proven efficacy in TRS. Clozapine has also been shown to have robust effects on preventing suicide (Meltzer, 2011) in this patient subgroup. However, although an estimated two-thirds of patients with treatment-resistant symptoms would respond beneficially to clozapine (Meltzer, 2011), it is still widely under-prescribed, in part due to the risk of serious adverse effects (De Fazio et al., 2015). One of these, affecting $0.38-0.8 \%$ of patients, is agranulocytosis, a potentially fatal condition in which there is a significant decrease in granulocytes, the most abundant form of which are neutrophils (Alvir et al., 1993). To minimize the risk of agranulocytosis, regular haematological monitoring is required for safe and effective treatment with clozapine. Clozapineinduced agranulocytosis is most likely to develop within the first 18 weeks of treatment (Atkin et al., 1996), during which time patients' white blood cell levels are measured weekly and treatment stopped if agranulocytosis is detected or monitoring shows a sustained but milder decrease of white blood cells (neutropenia). This need for intensive physical health monitoring is an additional burden for the use of clozapine, and has been indicated by service users as a reason for discontinuing treatment (Legge et al., 2016).

In contrast to hematological monitoring, measures of clozapine concentrations in plasma do not seem to be strong indicators of agranulocytosis onset (Hasegawa et al., 1994), which to this day is a manageable but unpredictable risk of this treatment (de Leon et al., 2020). However, potential associations between clozapine dose and ANC in those taking the drug remain poorly understood. Several mechanisms have been proposed in favor of the hypothesis that clozapine might have a subtle dose-dependent effect on ANC (Vaquero-Baez et al., 2019), which could, in turn, lead to neutropenia. One such mechanism is the production of a reactive nitrenium ion following the bioactivation of either clozapine or one of its primary metabolites (Liu and Uetrecht, 1995). In turn, this short-lived but highly reactive intermediate may act as a hapten leading to the destruction of neutrophils via an immune response, or it may disrupt neutrophil function by binding to crucial cellular proteins (Pirmohamed and Park, 1997). This effect could potentially be enhanced if an infection occurs, since inflammatory processes are known to increase clozapine plasma concentrations (de Leon et al., 2020; Siskind et al., 2020). Complicating the matter further is the fact that there is substantial variation of plasma clozapine concentration between individuals who have been prescribed the same dose; with modifying factors including age, weight, smoking and likely genomic variation affecting enzymes involved in clozapine metabolism (Olsson et al., 2015). It is important to better understand the link between clozapine doses, clozapine metabolism and neutrophil counts for several reasons. First, because it would allow for the evaluation of the effectiveness of interventions aimed at lessening the potential detrimental impacts of clozapine on the immune system, such as those proposed in response to the COVID-19 pandemic (Pandarakalam, 2020; Siskind et al., 2020). Additionally, in line with recent research on underrepresented populations (Legge et al., 2019), improved comprehension of the biology behind potential adverse effects could help with the refinement of current dosing and titration protocols, therefore increasing the number of TRS patients who can be safely prescribed the medication and benefit from its therapeutic effects.

There have been a small number of studies investigating the relationship between plasma concentrations of clozapine metabolites and blood dyscrasias, but results are so far inconclusive. In a study of 5 patients with confirmed agranulocytosis, plasma clozapine and norclozapine levels were below toxic ranges and not significantly different to 59 patients on clozapine who did not develop agranulocytosis (Hasegawa et al., 1994). Another study with a cohort of 37 schizophrenia patients taking clozapine reported no significant correlations between neutrophil count and serum clozapine and norclozapine levels (Oyewumi et al., 2002). However, a more recent observational study of 129 patients did report a significant positive correlation between norclozapine serum concentrations and ANC, with the same relationship being observed with the clozapine/ norclozapine ratio (Smith et al., 2017). Finally, and most recently, a study of 41 patients found a negative association between plasma clozapine concentration and neutrophil count, reporting an $R^{2}=0.447$ from multiple regression analyses (Vaquero-Baez et al., 2019). While all of these studies were arguably small for a cross-sectional design, the effect sizes reported in the latter could be conventionally considered as "large" (Cohen, 1992), which make them interesting candidates for further investigation. Motivated to test these findings on a larger cohort, we used similar statistical methods on the UK-based CLOZUK2 study of people with TRS (Pardiñas et al., 2018). We also extended the regression approach to incorporate genetic data, specifically genetic variants that have been recently found to be associated with clozapine metabolism (Pardiñas et al., 2019).

\section{METHODS}

\section{Samples, Neutrophil Count and Clozapine Concentration Data}

ANC, clozapine concentration and genetic data were obtained as part of the CLOZUK2 study. Full details of recruitment and quality control of the samples are provided in Pardiñas et al. (2018). Curation of ANC data and assessment of genetic ancestry are described in Legge et al. (2019), while curation of the 
TABLE 1 | Pharmacogenomic SNPs used in the regression analyses, and their association to clozapine metabolite concentrations in the GWAS from Pardiñas et al. (2019).

\begin{tabular}{lcccc}
\hline Phenotype/SNP & Minor Allele & Beta & s.e. & MAF $^{\mathbf{a}}$ \\
$\begin{array}{l}\text { Clozapine } \\
\text { rs2472297 }\end{array}$ & T & -0.089 & 0.013 & 27.94 \\
Norclozapine & & & & \\
rs61750900 & $T$ & -0.149 & 0.018 & 9.9 \\
rs2011425 & G & -0.112 & 0.019 & 8.65 \\
Metabolic ratio & & & & \\
rs61750900 & T & 0.212 & 0.012 & 9.9 \\
rs1126545 & T & 0.078 & 0.01 & 14.22 \\
& & & &
\end{tabular}

${ }^{a}$ Based on the European Subset of CLOZUK2 (see Pardiñas et al., 2019 for details).

longitudinal clozapine concentration data is described in Pardiñas et al. (2019).

\section{Inclusion Criteria}

The dataset was restricted to individuals of European ancestry who had a plasma clozapine measurement $(\mathrm{mg} / \mathrm{L})$ within 21 days of an ANC (cells $/ \mathrm{mm}^{3}$ ) measurement. For any individual who had more than one clozapine plasma concentration sample within that window, the measurement closest to the ANC was selected. The clozapine/norclozapine ratio ("metabolic ratio") was calculated for each individual, and anyone with a ratio greater than 3 and less than 0.5 was excluded $(n=19)$, in line with current recommendations to detect confounding factors such as drug interactions, as well as treatment non-adherence (Ellison and Dufresne, 2015). Finally, any individual prescribed clozapine for the treatment of psychosis in Parkinson's disease was also excluded $(n=1)$, leaving a final sample of 208 individuals.

\section{Statistical Analysis of Metabolite Data}

As a direct replication of Vaquero-Baez et al. (2019), Spearman correlations were computed to assess the bivariate relationships between plasma clozapine concentration, plasma norclozapine concentration, time on clozapine treatment, daily clozapine dose and ANC. Expanding on those analyses, multivariate linear regression models were used to assess the association between plasma clozapine concentration $(\mathrm{mg} / \mathrm{L})$ and ANC (cells $/ \mathrm{mm}^{3}$ ) accounting for eight additional covariates: norclozapine plasma concentration $(\mathrm{mg} / \mathrm{L})$, daily clozapine dose $(\mathrm{mg})$, time on clozapine treatment (days), time between clozapine concentration measurement and ANC measurement (days), time between clozapine dose and blood sampling (hours), sex (male/female), age (years) and age (in order to account for a possible non-linear effect of age on ANC; Prabhakar et al., 2009). Following on from this baseline model, the metabolic ratio and four pharmacogenomic variants (further details below) were added to refine our assessment of the relationship between clozapine and ANC and to identify potential mediators. All statistical analyses were conducted using R v4.0.2.

\section{Pharmacogenomic Analyses}

Four SNPs were selected for inclusion in these models, based on work conducted by Pardiñas et al. (2019), in which GWAS of clozapine levels, norclozapine levels, and clozapine/norclozapine metabolic ratio were conducted to identify pharmacogenomic variants affecting the metabolism of clozapine. Details of these SNPs can be seen in Table 1. Another SNP reported to be associated with clozapine concentration (rs28379954; Smith et al., 2020) was also considered for analysis, but was not included due to poor imputation quality in CLOZUK2, which led to missing data in 78/208 individuals in our sample.

\section{RESULTS}

Descriptive statistics for the final sample can be seen in Table 2 . This cohort represents long-term clozapine users, who at the time of sampling had ANC levels not indicative of neutropenia (ANC $\geq 1,500$ cells $/ \mathrm{mm}^{3}$ ) by clozapine monitoring guidelines (Nielsen et al., 2016).

\section{Correlation Analysis}

Mirroring the approach of Vaquero-Baez et al. (2019), Spearman's correlation statistics are reported in Table 3. None of the variables examined was significantly associated with ANC in this bivariate analysis.

\section{Regression Procedure}

Three regression models were considered: Model 1 includes clozapine plasma concentration and the eight covariates described above. Model 2 introduces the metabolic ratio as an additional covariate, following previous clozapine therapeutic drug monitoring studies (Rostami-Hodjegan et al., 2004; Couchman et al., 2013). Model 3 additionally incorporates four genetic variants associated with clozapine metabolism (Table 1).

TABLE 2 | Covariates used in the correlation and regression analyses, and their distribution in the CLOZUK2 sample described in this study.

Male, $N=147$

$0.47(0.28)$

$0.27(0.17)$

357 (136)

$40.3(13.2)$

$3.26(0.8)$

$3.1(1.2)$
Female, $N=61$

$0.51(0.32)$

$0.29(0.17)$

$322.2(134)$

$42.7(14.2)$

$3.21(1.1)$

$3.1(1.2)$ 
TABLE 3 | Spearman's rank correlations of several variables with ANC, reproducing the approach of Vaquero-Baez et al. (2019).

\begin{tabular}{lcc}
\hline Outcome & \multicolumn{2}{c}{ Lowest ANC } \\
\cline { 2 - 3 } & Correlation coefficient (rho) & $\boldsymbol{p}$ \\
\hline $\mathrm{CLZ}$ & -0.125 & 0.062 \\
DMC & -0.033 & 0.629 \\
Time on clozapine & -0.073 & 0.289 \\
Daily dose & -0.033 & 0.629
\end{tabular}

Effect size estimates of all covariates included in our ANC regression models are shown in Table 4.

\section{Model 1}

Both clozapine and norclozapine level were significantly associated with ANC; clozapine level was negatively associated $(\beta=-1.41, p=0.009)$ and a positive association was found for norclozapine $(\beta=1.77, p=0.049)$. For each $0.1 \mathrm{mg} / \mathrm{L}$ increase in plasma clozapine concentration, there was an associated decrease in ANC of 141 cells $/ \mathrm{mm}^{3}$, and for each $0.1 \mathrm{mg} / \mathrm{L}$ increase in norclozapine concentration, there was an increase in ANC of 177 cells $/ \mathrm{mm}^{3}$.

\section{Model 2}

With the addition of metabolic ratio in the model, the effect sizes of clozapine and norclozapine shifted toward zero, becoming nonsignificant likely because of collinearity with this covariate. Thus, their initial association appeared to be explained by their ratio, which was negatively associated with ANC $(\beta=-0.69, p=$ $0.021)$. For every unit increase in the clozapine/norclozapine ratio we estimated an associated decrease of 690 cells $/ \mathrm{mm}^{3}$ in ANC.

\section{Model 3}

Of the four SNPs included in the analysis, 3 were significantly associated with ANC; rs61750900_T $(\beta=-0.41, p=0.048)$, rs2011425_G $(\beta=0.45, p=0.026)$ and rs1126545_T $(\beta=0.33, p=$ $0.039)$. Each minor allele was associated with a decrease in ANC of 410 cells $/ \mathrm{mm}^{3}$ and increases of 450 cells $/ \mathrm{mm}^{3}$ and 330 cells/ $\mathrm{mm}^{3}$, respectively. In this model, the metabolic ratio remained significantly associated with a decrease in $\operatorname{ANC}(\beta=-0.54, p=0.035)$.

\section{DISCUSSION}

\section{Clozapine and Neutrophil Counts}

Our results demonstrate a relationship between clozapine concentration and ANC, which appeared to be mediated through the metabolic ratio of clozapine and its main metabolite norclozapine. As the ratio of clozapine/ norclozapine increased, ANC decreased, and this relationship appeared to be largely independent of clozapine dose. High metabolic ratios in the normal range (i.e. not suggestive of non-adherence; Ellison and Dufresne, 2015) could either be indicative of slow metabolism of clozapine or relatively rapid clearance of norclozapine, and have been previously associated to better cardiometabolic and cognitive outcomes (Costa-Dookhan et al., 2020). Regression results also highlighted a relationship with ANC of pharmacogenomic variants associated with the clozapine/norclozapine metabolic ratio, as well as norclozapine concentration. Interestingly, two of these variants (rs61750900 and rs2011425) are missense polymorphisms in UGT genes, which glucuronidize norclozapine and thereby facilitate its excretion, supporting the role of this metabolite in altering ANC (Erickson-Ridout et al., 2012). The third significantly associated SNP (rs1126545) is a missense variant located within CYP2C18. This gene, while not part of the current consensus clozapine metabolic pathway (Thorn et al., 2018), has also been shown to be able to participate in its bioactivation in vitro (Dragovic et al., 2013). The fourth SNP (rs2472297), not associated significantly with ANC in any of our analyses, is an intergenic variant located between two cytochrome P450 genes, CYP1A1 and CYP1A2. This marker, also commonly found in coffee consumption and caffeine metabolism GWAS, has been tentatively associated to the regulation of CYP1A2 activity (Pardiñas et al., 2019), which drives the primary

TABLE 4 | Results of the three regression analyses with ANC as outcome.

\begin{tabular}{|c|c|c|c|c|c|c|}
\hline \multirow[t]{2}{*}{ Variable } & \multicolumn{2}{|c|}{ Model 1} & \multicolumn{2}{|c|}{ Model 2} & \multicolumn{2}{|c|}{ Model 3} \\
\hline & Estimates & $p$ & Estimates & $p$ & Estimates & $p$ \\
\hline Clozapine concentration & -1.410 & 0.009 & 0.540 & 0.585 & 0.060 & 0.950 \\
\hline Norclozapine concentration & 1.770 & 0.049 & -1.450 & 0.385 & -0.570 & 0.738 \\
\hline Daily dose & 0.000 & 0.887 & 0.000 & 0.931 & 0.000 & 0.948 \\
\hline Gender [male] & 0.030 & 0.886 & 0.040 & 0.829 & 0.080 & 0.626 \\
\hline Days between clozapine/ANC measurements & -0.010 & 0.113 & -0.010 & 0.124 & -0.010 & 0.105 \\
\hline Age & 0.120 & 0.001 & 0.110 & 0.001 & 0.120 & 0.001 \\
\hline Age squared & -0.001 & 0.004 & -0.001 & 0.004 & -0.001 & 0.004 \\
\hline Time on clozapine & -0.001 & 0.036 & -0.150 & 0.044 & -0.001 & 0.020 \\
\hline Time between dose and sample & -0.050 & 0.105 & 0.060 & 0.080 & -0.050 & 0.122 \\
\hline Ratio & & & -0.690 & 0.021 & -0.540 & 0.035 \\
\hline rs2472297_T (clozapine) & & & & & -0.130 & 0.324 \\
\hline rs61750900_T (norclozapine + ratio) & & & & & -0.410 & 0.048 \\
\hline rs2011425_G (norclozapine) & & & & & 0.450 & 0.026 \\
\hline rs1126545_T (ratio) & & & & & 0.330 & 0.039 \\
\hline
\end{tabular}

Bold highlight indicates statistically significant effect sizes $(p<0.05)$. 
reaction transforming clozapine into norclozapine (Prior and Baker, 2003). While the exact biological effect that any these variants might have on neutrophil count itself cannot be inferred from our results, our analysis does highlight a number of potential avenues for further research.

\section{Replication of Previous Results}

Although our main findings replicate those of Vaquero-Baez et al. (2019), the effect sizes we observed were substantially smaller. Besides the effects of winner's curse (Kraft, 2008), and the use of a larger cohort (208 vs. 41), there are a number of reasons why this may be the case. Firstly, the original study was conducted in Mexico, and the individuals recruited are likely of different ancestry (Ruiz-Linares et al., 2014) to our cohort that was recruited in the UK and made up only of those of European ancestry as inferred from genetic analyses. It cannot be ruled out that the work of Vaquero-Baez et al. (2019) has uncovered a population- or ancestry-specific effect, although no literature exists at this time to support this. An increased prevalence of neutropenia has been found in individuals with schizophrenia of African ethnicity (Kelly et al., 2007), and "benign" (constitutional) neutropenia rates vary widely based on genetic ancestry (Haddy et al., 1999; Legge et al., 2019), but no specific risk has been found in Mexican, Latino or Native American people to date.

Another noteworthy difference is that the daily clozapine dose prescribed is substantially different between the two cohorts, as inferred from a comparison of the study descriptive statistics. In our study average clozapine doses were $348 \mathrm{mg} /$ day for males and $313 \mathrm{mg} /$ day for females, while Vaquero-Baez et al. (2019) reported average doses of $223 \mathrm{mg} /$ day for males and $105 \mathrm{mg} /$ day for females $\left(t\right.$-test $\left.p_{\text {male }}=1.31 \times 10^{-10} ; p_{\text {female }}=1.43 \times 10^{-6}\right)$. There was also a significant difference in the average time that the cohorts had been on treatment with clozapine, upwards of three years in our study with 3.26 years for males and 3.21 years for females, and less than one year in Vaquero-Baez et al. (2019) with 10 months for males and 6.5 months for females $\left(t\right.$-test $p_{\text {male }}=5.9$ $\times 10^{-3} ; p_{\text {female }}=6.18 \times 10^{-16}$ ). This might have contributed to the smaller effect size we observed between clozapine metabolites and ANC, as our sample represents individuals who are long-term clozapine users and therefore likely excludes the approximately $10 \%$ of people who, after a year of treatment, might go on to exhibit neutropenia or other immune-related adverse effects (Myles et al., 2018). However, the main associations we found across our successive tests suggest that clozapine might have sustained effects on ANC even in individuals without obvious hematological adverse effects. This is consistent with the rationale for the current practice of continued hematological monitoring of people being prescribed clozapine, and supports that additional measures to lower their risk of infections might indeed be warranted even in long term clozapine users (Siskind et al., 2020), for example ensuring their timely access to the annual influenza vaccine (Pandarakalam, 2020).

\section{Limitations}

There are a few limitations in this work that have to be noted. Although several covariates were used in the regression analyses, no data were available for the CLOZUK2 cohort for several factors that are known to affect clozapine metabolism, including concomitant medications (Singh et al., 2015), the use of tobacco (Smith and Mican, 2014) and the consumption of coffee (Raaska et al., 2004). Additionally, the cross-sectional nature of the ANC data available means there is no information regarding neutrophil trajectories throughout the individuals' time on treatment. Replicating this analysis in a longitudinal dataset would allow for better estimations of the magnitude of the detected associations and whether they vary along particular time or titration windows. This would additionally enable clarifying, through more sophisticated causal modeling, the exact relationship between clozapine metabolic ratio and ANC.

\section{CONCLUSION}

Increased blood clozapine concentration is associated with decreased ANC in people with treatment-resistant schizophrenia who are long-term recipients of clozapine, even if they have not developed agranulocytosis or neutropenia during their treatment. Further investigation suggests this relationship is mediated by the clozapine/norclozapine metabolic ratio, with higher values (increased levels of clozapine proportional to norclozapine) associated with lower ANC. Furthermore, these effects can be partially explained by common genetic variants, some of which have a functional impact on enzymes involved in norclozapine glucuronidation (rs61750900 and rs2011425). Investigating these effects in a longitudinal cohort could shed further light on the relationship between clozapine and neutrophils, potentially offering biological insights. A fuller mechanistic understanding of the linkage between clozapine and ANC might allow improving the wellbeing of individuals taking clozapine by supporting targeted interventions such as prioritizing seasonal vaccinations. Additional research might also lead to the elucidation of appropriate trials to assess the incorporation of genetic variants into clozapine monitoring and/or titration protocols, thus tapping into currently unrecognized but potentially valuable sources of information to improve clozapine safety at all stages of treatment.

\section{DATA AVAILABILITY STATEMENT}

The data analyzed in this study is subject to the following licenses/ restrictions: To comply with the ethical and regulatory framework of the CLOZUK project, access to individual-level data requires a collaboration agreement with Cardiff University. Requests to access these datasets should be directed to Prof. James T. R. Walters (WaltersJT@cardiff.ac.uk).

\section{ETHICS STATEMENT}

The CLOZUK project was reviewed and approved by the UK Multicentre Research Ethics Committee (ref. 10/WSE02/15). Written informed consent for participation was not required for this study in accordance with the national legislation and the institutional requirements. 


\section{AUTHOR CONTRIBUTIONS}

SL, JW, and AP conceived and designed the study. SL, MN, AK, JJ, and $\mathrm{MH}$ contributed and/or curated data. IW performed all statistical analyses under the supervision of AP. IW, JW, and AP interpreted results. IW, SL, LM, MO, MO'D, JW, and AP participated in the primary drafting of the manuscript. All authors had the opportunity to review and comment on the manuscript, and all approved the final manuscript.

\section{FUNDING}

Academy of Medical Sciences Springboard award to AFP (SBF005\1083).

Owen, O'Donovan, and Walters are supported by a collaborative research grant from Takeda Pharmaceuticals

\section{REFERENCES}

Alvir, J. M. J., Lieberman, J. A., Safferman, A. Z., Schwimmer, J. L., and Schaaf, J. A. (1993). Clozapine-induced agranulocytosis-incidence and risk factors in the United States. N. Engl. J. Med. 329 (3), 162-167. doi:10.1056/ nejm199307153290303

Atkin, K., Kendall, F., Gould, D., Freeman, H., Lieberman, J., and O’sullivan, D. (1996). Neutropenia and agranulocytosis in patients receiving clozapine in the UK and Ireland. Br. J. Psychiatry 169 (4), 483-488. doi:10.1192/bjp.169. 4.483

Cohen, J. (1992). A power primer. Psychol. Bull. 112 (1), 155-159. doi:10.1037/ 0033-2909.112.1.155

Costa-Dookhan, K. A., Agarwal, S. M., Chintoh, A., Tran, V. N., Stogios, N., Ebdrup, B. H., et al. (2020). The clozapine to norclozapine ratio: a narrative review of the clinical utility to minimize metabolic risk and enhance clozapine efficacy. Expert Opin. Drug Saf. 19 (1), 43-57. doi:10.1080/14740338.2020. 1698545

Couchman, L., Bowskill, S. V. J., Handley, S., Patel, M. X., and Flanagan, R. J. (2013). Plasma clozapine and norclozapine in relation to prescribed dose and other factors in patients aged < 18 years: data from a therapeutic drug monitoring service, 1994-2010. Early Interv Psychiatry 7 (2), 122-130. doi:10.1111/j.1751-7893.2012.00374.x

De Fazio, P., Gaetano, R., Caroleo, M., Cerminara, G., Maida, F., Bruno, A., et al. (2015). Rare and very rare adverse effects of clozapine. Neuropsychiatr. Dis. Treat. 11, 1995-2003. doi:10.2147/ndt.s83989

De leon, J., Ruan, C. J., Schoretsanitis, G., and De las cuevas, C. (2020). A rational use of clozapine based on adverse drug reactions, pharmacokinetics, and clinical pharmacopsychology. Psychother. Psychosom. 89 (4), 200-214. doi:10.1159/ 000507638

Dragovic, S., Gunness, P., Ingelman-Sundberg, M., Vermeulen, N. P. E., and Commandeur, J. N. M. (2013). Characterization of human cytochrome P450s involved in the bioactivation of clozapine. Drug Metab. Dispos. 41 (3), 651-658. doi:10.1124/dmd.112.050484

Ellison, J. C., and Dufresne, R. L. (2015). A review of the clinical utility of serum clozapine and norclozapine levels. Ment. Health Clinician 5 (2), 68-73. doi:10. 9740/mhc.2015.03.068

Erickson-Ridout, K. K., Sun, D., and Lazarus, P. (2012). Glucuronidation of the second-generation antipsychotic clozapine and its active metabolite $\mathrm{N}$-desmethylclozapine. Potential importance of the UGT1A1 A(TA)7TAA and UGT1A4 L48V polymorphisms. Pharmacogenet. Genomics. 22 (8), 561-576. doi:10.1097/fpc.0b013e328354026b

Haddy, T. B., Rana, S. R., and Castro, O. (1999). Benign ethnic neutropenia: what is a normal absolute neutrophil count?. J. Lab. Clin. Med. 133 (1), 15-22. doi:10. 1053/lc.1999.v133.a94931
(Takeda played no part in the conception, design, implementation, or interpretation of this study)

\section{ACKNOWLEDGMENTS}

JTRW is supported by a UK Medical Research Council Mental Health Data Pathfinder grant (MC-PC-17212). AFP is supported by an Academy of Medical Sciences "Springboard" award (SBF005\1083). The CLOZUK project was supported by the following grants to Cardiff University: European Union's Seventh Framework Programme (279227), Medical Research Council Centre (MR/L010305/1), Program (G0800509), and Project (MR/L011794/1). We acknowledge Lesley Bates, Catherine Bresner and Lucinda Hopkins for laboratory sample management at Cardiff University.

Hasegawa, M., Cola, P. A., and Meltzer, H. Y. (1994). Plasma clozapine and desmethylclozapine levels in clozapine-induced agranulocytosis. Neuropsychopharmacol 11 (1), 45-47. doi:10.1038/npp.1994.34

Howes, O. D., Mccutcheon, R., Agid, O., De Bartolomeis, A., Van Beveren, N. J. M., Birnbaum, M. L., et al. (2017). Treatment-resistant schizophrenia: treatment response and resistance in psychosis (TRRIP) working group consensus guidelines on diagnosis and terminology. Am. J. Psychiatry. 174 (3), 216-229. doi:10.1176/appi.ajp.2016.16050503

Kelly, D. L., Kreyenbuhl, J., Dixon, L., Love, R. C., Medoff, D., and Conley, R. R. (2007). Clozapine underutilization and discontinuation in African Americans due to leucopenia. Schizophr Bull. 33 (5), 1221-1224. doi:10. $1093 / \mathrm{schbul} / \mathrm{sbl068}$

Kraft, P. (2008). Curses-winner's and otherwise-in genetic epidemiology. Epidemiology 19 (5), 649-651. doi:10.1097/ede.0b013e318181b865

Legge, S. E., Hamshere, M., Hayes, R. D., Downs, J., O’donovan, M. C., Owen, M. J., et al. (2016). Reasons for discontinuing clozapine: a cohort study of patients commencing treatment. Schizophr Res. 174 (1-3), 113-119. doi:10.1016/j. schres.2016.05.002

Legge, S. E., Pardiñas, A. F., Helthuis, M., Jansen, J. A., Jollie, K., Knapper, S., et al. (2019). A genome-wide association study in individuals of African ancestry reveals the importance of the Duffy-null genotype in the assessment of clozapine-related neutropenia. Mol. Psychiatry 24 (3), 328-337. doi:10.1038/ s41380-018-0335-7

Liu, Z. C., and Uetrecht, J. P. (1995). Clozapine is oxidized by activated human neutrophils to a reactive nitrenium ion that irreversibly binds to the cells. J. Pharmacol. Exp. Ther. 275 (3), 1476-1483.

Mcgrath, J., Saha, S., Chant, D., and Welham, J. (2008). Schizophrenia: a concise overview of incidence, prevalence, and mortality. Epidemiol. Rev. 30, 67-76. doi:10.1093/epirev/mxn001

Meltzer, H. Y. (2011). Role of clozapine in treatment-resistant schizophrenia. Adv. Biol. Psychiatry 26, 114-128. doi:10.1159/000319812

Myles, N., Myles, H., Xia, S., Large, M., Kisely, S., Galletly, C., et al. (2018). Metaanalysis examining the epidemiology of clozapine-associated neutropenia. Acta Psychiatr. Scand. 138 (2), 101-109. doi:10.1111/acps.12898

Nielsen, J., Young, C., Ifteni, P., Kishimoto, T., Xiang, Y.-T., Schulte, P. F. J., et al. (2016). Worldwide differences in regulations of clozapine use. CNS Drugs 30 (2), 149-161. doi:10.1007/s40263-016-0311-1

Olsson, E., Edman, G., Bertilsson, L., Hukic, D. S., Lavebratt, C., Eriksson, S. V., et al. (2015). Genetic and clinical factors affecting plasma clozapine concentration. Prim. Care Companion CNS Disord. 17 (1). doi:10.4088/PCC. $14 \mathrm{~m} 01704$

Oyewumi, L. K., Cernovsky, Z. Z., Freeman, D. J., and Streiner, D. L. (2002) Relation of blood counts during clozapine treatment to serum concentrations of clozapine and nor-clozapine. Can. J. Psychiatry 47 (3), 257-261. doi:10.1177/ 070674370204700306 
Pandarakalam, J. P. (2020). Revisiting clozapine in a setting of COVID-19. Am. J. Psychiatry Neurosci. 8 (3), 50-58. doi:10.11648/j.ajpn.20200803.12

Pardiñas, A. F., Holmans, P., Holmans, P., Pocklington, A. J., Escott-Price, V., Ripke, S., et al. (2018). Common schizophrenia alleles are enriched in mutationintolerant genes and in regions under strong background selection. Nat. Genet. 50 (3), 381-389. doi:10.1038/s41588-018-0059-2

Pardiñas, A. F., Nalmpanti, M., Pocklington, A. J., Legge, S. E., Medway, C., King, A., et al. (2019). Pharmacogenomic variants and drug interactions identified through the genetic analysis of clozapine metabolism. Am. J. Psychiatry. 176 (6), 477-486. doi:10.1176/appi.ajp.2019.18050589

Pirmohamed, M., and Park, K. (1997). Mechanism of clozapine-induced agranulocytosis : current status of research and implications for drug development. CNS Drugs 7 (2), 139-158. doi:10.2165/00023210-19970702000005

Prabhakar, M., Ershler, W. B., and Longo, D. L. (2009). Bone marrow, thymus and blood: changes across the lifespan. Aging Health 5 (3), 385-393. doi:10.2217/ ahe.09.31

Prior, T. I., and Baker, G. B. (2003). Interactions between the cytochrome P450 system and the second-generation antipsychotics. J. Psychiatry Neurosci. 28 (2), 99-112.

Raaska, K., Raitasuo, V., Laitila, J., and Neuvonen, P. J. (2004). Effect of caffeinecontaining versus decaffeinated coffee on serum clozapine concentrations in hospitalised patients. Pharmacol. Toxicol. 94 (1), 13-18. doi:10.1111/j.17427843.2004.pto940104.x

Rostami-Hodjegan, A., Amin, A. M., Spencer, E. P., Lennard, M. S., Tucker, G. T., and Flanagan, R. J. (2004). Influence of dose, cigarette smoking, age, sex, and metabolic activity on plasma clozapine concentrations: a predictive model and nomograms to aid clozapine dose adjustment and to assess compliance in individual patients. J. Clin. Psychopharmacol. 24 (1), 70-78. doi:10.1097/01.jcp. $0000106221.36344 .4 \mathrm{~d}$

Ruiz-Linares, A., Adhikari, K., Acuña-Alonzo, V., Quinto-Sanchez, M., Jaramillo, C., Arias, W., et al. (2014). Admixture in Latin America: geographic structure, phenotypic diversity and self-perception of ancestry based on 7,342 individuals. PLoS Genet. 10 (9), e1004572. doi:10.1371/journal.pgen.1004572

Singh, H., Dubin, W. R., and Kaur, S. (2015). Drug interactions affecting clozapine levels. J. Psychiatr. Intensive Care 11 (01), 52-65. doi:10.1017/ s1742646413000332
Siskind, D., Honer, W. G., Clark, S., Correll, C. U., Hasan, A., Howes, O., et al. (2020). Consensus statement on the use of clozapine during the COVID-19 pandemic. J. Psychiatry Neurosci. 45 (3), 222-223. doi:10.1503/jpn.200061

Smith, R. L., Haslemo, T., Andreassen, O. A., Eliasson, E., Dahl, M. L., Spigset, O., et al. (2017). Correlation between serum concentrations of $\mathrm{N}$-desmethylclozapine and granulocyte levels in patients with schizophrenia: a retrospective observational study. CNS Drugs. 31 (11), 991-997. doi:10.1007/ s40263-017-0469-1

Smith, R. L., O'connell, K., Athanasiu, L., Djurovic, S., Kringen, M. K., Andreassen, O. A., et al. (2020). Identification of a novel polymorphism associated with reduced clozapine concentration in schizophrenia patients-a genome-wide association study adjusting for smoking habits. Transl. Psychiatry 10 (1), 198. doi:10.1038/s41398-020-01061-4

Smith, T., and Mican, L. (2014). What to do when your patient who takes clozapine enters a smoke-free facility. Curr. Psychiatry 13 (5), 47-48.

Thorn, C. F., Müller, D. J., Altman, R. B., and Klein, T. E. (2018). PharmGKB summary. Pharmacogenet. Genom. 28, 214-222. doi:10.1097/fpc. 0000000000000347

Vaquero-Baez, M., Díaz-Ruíz, A., Tristán-López, L., Aviña-Cervantes, C., Torner, C., Ramírez-Bermúdez, J., et al. (2019). Clozapine and desmethylclozapine: correlation with neutrophils and leucocytes counting in Mexican patients with schizophrenia. BMC Psychiatry 19 (1), 295. doi:10.1186/s12888-019-2286-1

Conflict of Interest: MH and JJ were employed by the company Leyden Delta. AK was employed by the company Magna Laboratories.

The remaining authors declare that the research was conducted in the absence of any commercial or financial relationships that could be construed as a potential conflict of interest.

Copyright () 2021 Willcocks, Legge, Nalmpanti, Mazzeo, King, Jansen, Helthuis, Owen, O'Donovan, Walters and Pardiñas. This is an open-access article distributed under the terms of the Creative Commons Attribution License (CC BY). The use, distribution or reproduction in other forums is permitted, provided the original author(s) and the copyright owner(s) are credited and that the original publication in this journal is cited, in accordance with accepted academic practice. No use, distribution or reproduction is permitted which does not comply with these terms. 